\title{
A Frobenius characterization of finite projective dimension over complete intersections
}

\author{
Claudia Miller ${ }^{\star}$ \\ Department of Mathematics, University of Michigan, Ann Arbor, MI 48109, USA \\ (e-mail: clamille@math.lsa.umich.edu)
}

Received June 22, 1998; in final form October 13, 1998

\begin{abstract}
Let $M$ be a module of finite length over a complete intersection $(R, m)$ of characteristic $p>0$. We characterize the property that $M$ has finite projective dimension in terms of the asymptotic behavior of a certain length function defined using the Frobenius functor. This may be viewed as the converse to a theorem of S. Dutta. As a corollary we get that, in a complete intersection $(R, m)$, an $m$-primary ideal $I$ has finite projective dimension if and only if its Hilbert-Kunz multiplicity equals the length of $R / I$.
\end{abstract}

\section{Introduction and preliminaries}

Throughout our discussion all rings are commutative, Noetherian and contain a field of characteristic $p$. Let $F(-)$ denote the Frobenius functor and $\ell$ the length function. Our main result is the following theorem.

Theorem 2.1 Let $(R, m, k)$ be a Noetherian local ring of dimension $d$ which is a complete intersection and $M$ an $R$-module of finite length. Then

a) $\ell\left(F_{R}^{n}(M)\right) \geq \ell(M) \cdot p^{\text {nd }}$ for all $n \in \mathbb{N}$.

b) The sequence $\left\{p^{-n d} \ell\left(F_{R}^{n}(M)\right)\right\}$ is monotone non-decreasing.

c) The following are equivalent:

i) $M$ has finite projective dimension

ii) $\ell\left(F^{n}(M)\right)=\ell(M) p^{n d}$ for all $n \geq 0$

\footnotetext{
* This research was carried out while the author was supported by a research grant from the UIUC Campus Research Board of the University of Illinois under the supervision of S. Dutta.
} 
iii) $\lim _{n \rightarrow \infty} \frac{\ell\left(F^{n}(M)\right)}{p^{n d}}=\ell(M)$.

Statements $a$ ) and $c$ ), $i) \Rightarrow i i$ ), are due to S. Dutta ([D1, Theorem 1.9]). The proof of Theorem 2.1 depends greatly on the fact stated in part $a$ ). Using techniques developed in [D1] we prove the converse to Dutta's theorem, thus obtaining a characterization of modules of finite projective dimension over complete intersections.

Our result generalizes a characterization of regular local rings of characteristic $p$ due to E. Kunz [K1, Theorem 3.3]. His other characterization of regularity stated in the same theorem, namely that the Frobenius morphism is flat, was generalized by results of Peskine and Szpiro and of Herzog. We record these results since we will be using them later.

Theorem 1.1 ([PS, Theorem 1.7]) Suppose that $M$ is a finitely generated module over a ring of characteristic $p$. If $M$ has finite projective dimension over $R$, then $\operatorname{Tor}_{i}^{R}\left(M,{ }^{n} R\right)=0$ for all $i>0$ and $n>0$.

Theorem 1.2 ([He, Theorem 3.1]) Suppose that $M$ is a finitely generated $R$-module. If there exists an unbounded sequence of positive integers $\left(n_{k}\right)$ for which $\operatorname{Tor}_{i}^{R}\left(M,{ }^{f^{n}} R\right)=0$ for all $i>0$, then $M$ has finite projective dimension.

In Sect. 2 we give the proof of Theorem 2.1 and then specialize it to a statement about the Hilbert-Kunz multiplicity of ideals in complete intersection rings in Corollary 2.3. In Sect. 3 we prove a similar result for cyclic modules over certain one-dimensional Cohen-Macaulay rings, and we give an example to illustrate that such results cannot be expected to hold over general Cohen-Macaulay rings.

We end this section with a review of relevant terminology and some properties of the Frobenius functor. The Frobenius endomorphism $f$ of a ring $R$ of characteristic $p>0$ is defined by $f(r)=r^{p}$. Let ${ }^{f} R$ denote $R$ as an $R$ - $R$-bimodule, with multiplication on the left via $f$ and on the right in the usual way. The assignment $F_{R}(M)=M \otimes_{R}{ }^{f} R$ defines a functor on the category of $R$-modules to itself, where the $R$-module structure of $F_{R}(M)$ is via the righthand factor. We will usually write $F(M)$ when the underlying ring is understood. We let $F^{n}$ denote $n$ iterations of the Frobenius functor $F$. In fact, $F^{n}(M)$ is isomorphic to $M \otimes_{R} f^{n} R$, where $f^{n}$ denotes $n$ iterations of $f$ and $f^{n} R$ denotes $R$ as an $R$-R-bimodule, with multiplication on the left via $f^{n}$ and on the right in the usual way. If $M$ is a finitely generated $R$-module, then $F_{R}(M)$ is also a finitely generated $R$-module, and both have the same support ([PS], Proposition 1.5):

$$
\operatorname{Supp}_{R}\left(F_{R}(M)\right)=\operatorname{Supp}_{R}(M) .
$$


The behavior of the Frobenius functor on free and cyclic modules is as follows: if $s$ is a positive integer and $I$ is an ideal of $R$, then

$$
\begin{gathered}
F_{R}^{n}\left(R^{s}\right) \cong R^{s} \\
F_{R}^{n}(R / I) \cong R / I^{\left[p^{n}\right]},
\end{gathered}
$$

where $I^{\left[p^{n}\right]}$ denotes the ideal generated by the $p^{n}$-th powers of the generators of $I$. If $S \rightarrow R$ is a ring homomorphism and $M$ is an $S$-module, then $F_{S}^{n}(M) \otimes_{S} R \cong F_{R}^{n}\left(M \otimes_{S} R\right)$. If $M$ and $N$ are $R$-modules, then $F^{n}(M) \otimes_{R} F^{n}(N) \cong F^{n}\left(M \otimes_{R} N\right)$. In particular, if a sequence of elements $\underline{x}$ annihilates an $R$-module $M$, then $F_{R}^{n}(M) \otimes_{R} R / \underline{x}^{p^{n d}} \cong F_{R}^{n}(M)$.

\section{Proofs of the main results}

For completeness, we include the proofs of Dutta's of statements $a$ ) and $c), i) \Rightarrow i i)$ in the proof of the Theorem below.

Theorem 2.1 Let $(R, m, k)$ be a Noetherian local ring of dimension $d$ which is a complete intersection and $M$ an R-module of finite length. Then

a) $\ell\left(F_{R}^{n}(M)\right) \geq \ell(M) \cdot p^{\text {nd }}$ for all $n \in \mathbb{N}$.

b) The sequence $\left\{p^{-n d} \ell\left(F_{R}^{n}(M)\right)\right\}$ is monotone non-decreasing.

c) The following are equivalent:

i) $M$ has finite projective dimension

ii) $\ell\left(F^{n}(M)\right)=\ell(M) p^{n d}$ for all $n \geq 0$

iii) $\lim _{n \rightarrow \infty} \frac{\ell\left(F^{n}(M)\right)}{p^{n d}}=\ell(M)$.

Proof We begin with some arguments from [D1] which will be used in several parts of the proof. By hypothesis, $R$ can be written as $R=S /\left(x_{1}, x_{2}\right.$, $\left.\ldots, x_{t}\right)$, where $S$ is a regular local ring and $x_{1}, x_{2}, \ldots, x_{t}$ are elements of $S$ which form a regular sequence. Since the dimension of $R$ is $d$, the dimension of $S$ must be $d+t$. Let $\underline{x}$ denote the ideal $\left(x_{1}, x_{2}, \ldots, x_{t}\right)$, and let $\underline{x}^{p^{n}}$ denote the ideal $\left(x_{1}^{p^{n}}, x_{2}^{p^{n}}, \ldots, x_{t}^{p^{n}}\right)$. Consider a filtration of $S / \underline{x}^{p^{n}}$ by $p^{n t}$ copies of $S / \underline{x}$, that is, a set of short exact sequences of the form:

$$
\begin{gathered}
0 \rightarrow K_{1} \rightarrow S / \underline{x}^{p^{n}} \rightarrow S / \underline{x} \rightarrow 0 \\
0 \rightarrow K_{2} \rightarrow K_{1} \rightarrow S / \underline{x} \rightarrow 0 \\
\vdots \\
0 \rightarrow S / \underline{x} \rightarrow K_{p^{n t}-2} \rightarrow S / \underline{x} \rightarrow 0 .
\end{gathered}
$$

Tensoring the above sequences over $S$ with $F_{S}^{n}(M)$ gives a set of long exact sequences of $\operatorname{Tor}_{i}^{S}\left(F_{S}^{n}(M),-\right), i \geq 0$. Using the isomorphisms 
$F_{S}^{n}(M) \otimes_{S} S / \underline{x}^{p^{n}} \cong F_{S}^{n}(M)$ and $F_{S}^{n}(M) \otimes_{S} S / \underline{x} \cong F_{R}^{n}(M)$, one can write these sequences as follows:

$$
\begin{gathered}
\stackrel{\delta_{1}}{\longrightarrow} F_{S}^{n}(M) \otimes_{S} K_{1} \rightarrow F_{S}^{n}(M) \rightarrow F_{R}^{n}(M) \rightarrow 0 \\
\stackrel{\delta_{2}}{\longrightarrow} F_{S}^{n}(M) \otimes_{S} K_{2} \rightarrow F_{S}^{n}(M) \otimes_{S} K_{1} \rightarrow F_{R}^{n}(M) \rightarrow 0 \\
\vdots \\
\stackrel{\delta_{p^{n t}-1}}{\longrightarrow} F_{R}^{n}(M) \rightarrow F_{S}^{n}(M) \otimes_{S} K_{p^{n t}-1} \rightarrow F_{R}^{n}(M) \rightarrow 0 .
\end{gathered}
$$

Computing lengths gives the inequalities

$$
\begin{aligned}
\ell(M) p^{n(t+d)} & =\ell\left(F_{S}^{n}(M)\right) \\
& \leq \ell\left(F_{R}^{n}(M)\right)+\ell\left(F_{S}^{n}(M) \otimes K_{1}\right) \\
& \leq \ell\left(F_{R}^{n}(M)\right)+\ell\left(F_{R}^{n}(M)\right)+\ell\left(F_{S}^{n}(M) \otimes K_{2}\right) \\
& \cdots \\
& \leq p^{n t} \ell\left(F_{R}^{n}(M)\right)
\end{aligned}
$$

where the first line holds because $S$ is a regular local ring.

Part a) follows immediately from the above inequalities. Part b) follows from part a) since for all $n \geq 0$

$$
\ell\left(F^{n+1}(M)\right)=\ell\left(F\left(F^{n}(M)\right)\right) \geq p^{d} \ell\left(F^{n}(M)\right)
$$

by a) and thus

$$
\frac{\ell\left(F^{n+1}(M)\right)}{p^{(n+1) d}} \geq \frac{\ell\left(F^{n}(M)\right)}{p^{n d}}
$$

for all $n \geq 0$.

For c), we first note that the equivalence of ii) and iii) is immediate from b). As in [D1], the implication i) $\Rightarrow$ ii) is a consequence of a) as follows. Suppose $M$ has finite projective dimension. Let $y$ be a maximal $R$-sequence in the annihilator of $M$, and consider a short exact sequence

$$
0 \rightarrow Q \rightarrow(R /(\underline{y}))^{k} \rightarrow M \rightarrow 0 .
$$

Since $\operatorname{Tor}_{i}\left(M, f^{n} R\right)=0$ for all $i>0$ and $n>0$ by Theorem 1.1, tensoring this sequence with ${ }^{f^{n}} R$ gives an exact sequence

$$
0 \rightarrow F^{n}(Q) \rightarrow F^{n}\left((R /(\underline{y}))^{k}\right) \rightarrow F^{n}(M) \rightarrow 0 .
$$

Computing lengths, we get that

$$
\frac{\ell\left(F^{n}(M)\right)}{p^{n d}}+\frac{\ell\left(F^{n}(Q)\right)}{p^{n d}}=\frac{\ell\left(F^{n}\left((R /(\underline{y}))^{k}\right)\right)}{p^{n d}} .
$$


Since $y$ is an $R$-sequence, the righthand side is just $k \ell(R /(y))$, which equals $\ell(M)+\ell(Q)$ by the original short exact sequence. We therefore have

$$
\frac{\ell\left(F^{n}(M)\right)}{p^{n d}}+\frac{\ell\left(F^{n}(Q)\right)}{p^{n d}}=\ell(M)+\ell(Q) .
$$

Since $\ell\left(F^{n}(M)\right) / p^{n d} \geq \ell(M)$ and $\ell\left(F^{n}(Q)\right) / p^{n d} \geq \ell(Q)$, this forces $\ell\left(F^{n}(M)\right) / p^{n d}=\ell(M)$.

For the other implication, we note that if $\ell\left(F^{n}(M)\right)=\ell(M) p^{n d}$ for all $n \geq 0$ then the inequalities $(* *)$ derived earlier become

$$
\ell(M) p^{n(t+d)} \leq p^{n t} \ell\left(F_{R}^{n}(M)\right)=\ell(M) p^{n(t+d)} .
$$

So, all the inequalities in $(* *)$ must actually be equalities. This means that each of the maps $\delta_{i}$ in the long exact sequences $(*)$ must be zero. In particular $\delta_{1}=0$, i.e., we have a surjective map

$$
\psi_{1}: \operatorname{Tor}_{1}^{S}\left(F_{S}^{n}(M), S / \underline{x}^{p^{n}}\right) \rightarrow \operatorname{Tor}_{1}^{S}\left(F_{S}^{n}(M), S / \underline{x}\right)
$$

of the long exact sequence.

Let $T$ be a finitely generated $S$-module. We use $\psi_{T}$ to denote the natural map

$$
\operatorname{Tor}_{1}^{S}\left(F_{S}^{n}(T), S / \underline{x}^{p^{n}}\right) \rightarrow \operatorname{Tor}_{1}^{S}\left(F_{S}^{n}(T), S / \underline{x}\right)
$$

induced by the surjection $S / \underline{x}^{p^{n}} \rightarrow S / \underline{x}$. Letting $F$. be a free resolution of $T$ over $S$ and using the fact that the Frobenius functor $F_{S}(-)$ is exact since $S$ is regular, we have isomorphisms

$$
\begin{aligned}
& \operatorname{Tor}_{1}^{S}\left(F_{S}^{n}(T), S / \underline{x}^{p^{n}}\right) \cong \mathrm{H}_{1}\left(F_{S}^{n}(F .) \otimes_{S} S / \underline{x}^{p^{n}}\right) \\
\cong & \mathrm{H}_{1}\left(F_{S}^{n}(F .) \otimes_{S} F_{S}^{n}(S / \underline{x})\right) \cong \mathrm{H}_{1}\left(F_{S}^{n}\left(F . \otimes_{S} S / \underline{x}\right)\right) \\
\cong & F_{S}^{n}\left(\mathrm{H}_{1}\left(F . \otimes_{S} S / \underline{x}\right)\right) \cong F_{S}^{n}\left(\operatorname{Tor}_{1}^{S}(T, S / \underline{x})\right) .
\end{aligned}
$$

Thus we have a map

$$
F_{S}^{n}\left(\operatorname{Tor}_{1}^{S}(T, S / \underline{x})\right) \rightarrow \operatorname{Tor}_{1}^{S}\left(F_{S}^{n}(T), S / \underline{x}\right)
$$

which we will also call $\psi_{T}$. Since $\underline{x}$ annihilates the image, the map $\psi_{T}$ factors through a map $\theta_{T}$ :

$$
F_{R}^{n}\left(\operatorname{Tor}_{1}^{S}(T, S / \underline{x})\right)=F_{S}^{n}\left(\operatorname{Tor}_{1}^{S}(T, S / \underline{x})\right) \otimes_{S} R \rightarrow \operatorname{Tor}_{1}^{S}\left(F_{S}^{n}(T), S / \underline{x}\right) .
$$

Notice that by construction $\theta_{T}$ is functorial in $T$. Furthermore, $\theta_{M}$ is surjective since $\psi_{1}=\psi_{M}$ is surjective as shown above. More generally, for any module $T$ of finite length and finite projective dimension, since $\ell\left(F^{n}(T)\right)=\ell(T) p^{n d}$ for all $n>0$, the same argument shows that $\theta_{T}$ is surjective. 
We now argue as in the proof of Theorem 2.2 in [D1] to obtain that $\operatorname{Tor}_{1}^{R}\left(M, f^{n} R\right)=0$ for all $n>0$. Let $y$ in $S$ be a maximal $R$-sequence in the annihilator $\operatorname{Ann}_{S} M$, and consider an exact sequence

$$
0 \rightarrow K \rightarrow(S /(\underline{x}, \underline{y}))^{k} \rightarrow M \rightarrow 0 \text {. }
$$

Tensoring with $S / \underline{x}$ one obtains

$$
0 \rightarrow \operatorname{Tor}_{1}^{S}(K, S / \underline{x}) \rightarrow \operatorname{Tor}_{1}^{S}\left((S /(\underline{x}, \underline{y}))^{k}, S / \underline{x}\right) \rightarrow \operatorname{Tor}_{1}^{S}(M, S / \underline{x}) \rightarrow 0,
$$

which is exact: using the Koszul resolution of $S / \underline{x}$ over $S$, one sees that each term is just $t$ copies of the corresponding term in the first exact sequence and the maps are the obvious ones. Applying the Frobenius functor over $R$ and using the functoriality of $\theta_{T}$ in $T$ gives a commutative diagram:

$$
\begin{aligned}
& F_{R}^{n}\left(\operatorname{Tor}_{1}^{S}\left((S /(\underline{x}, \underline{y}))^{k}, S / \underline{x}\right)\right) \stackrel{\pi}{\longrightarrow} F_{R}^{n}\left(\operatorname{Tor}_{1}^{S}(M, S / \underline{x})\right) \\
& \downarrow \theta_{(S /(\underline{x}, \underline{y}))^{k}} \downarrow \theta_{M} \\
& \operatorname{Tor}_{1}^{S}\left(F_{S}^{n}(S /(\underline{x}, \underline{y})), S / \underline{x}\right) \quad \stackrel{\mu}{\longrightarrow} \operatorname{Tor}_{1}^{S}\left(F_{S}^{n}(M), S / \underline{x}\right)
\end{aligned}
$$

where the second row is part of the long exact sequence obtained by tensoring the sequence

$$
0 \rightarrow F_{S}^{n}(K) \rightarrow F_{S}^{n}(S /(\underline{x}, \underline{y}))^{k} \rightarrow F_{S}^{n}(M) \rightarrow 0
$$

with $S / \underline{x}=R$. Since $\theta_{M}$ and $\pi$ are surjective, so is $\mu$. Therefore the first connecting homomorphism $\delta: \operatorname{Tor}_{1}^{S}\left(F_{S}^{n}(M), S / \underline{x}\right) \rightarrow F_{R}^{n}(K)$ of the long exact sequence is the zero map, and so the sequence

$$
0 \rightarrow F_{R}^{n}(K) \rightarrow F_{R}^{n}(S /(\underline{x}, \underline{y}))^{k} \rightarrow F_{R}^{n}(M) \rightarrow 0
$$

is exact. This implies that $\operatorname{Tor}_{1}^{R}\left(M, f^{n} R\right)=0$ since by Theorem 1.1 we have that $\operatorname{Tor}_{1}^{R}\left((S /(\underline{x}, \underline{y}))^{k}, f^{n} R\right)=0$.

Since the sequence

$$
0 \rightarrow F_{R}^{n}(K) \rightarrow F_{R}^{n}(R /(\underline{y}))^{k} \rightarrow F_{R}^{n}(M) \rightarrow 0
$$

is exact, we also obtain that

$$
\begin{aligned}
\ell\left(F_{R}^{n}(K)\right) & =\ell\left(F_{R}^{n}(R /(\underline{y}))^{k}\right)-\ell\left(F_{R}^{n}(M)\right) \\
& =\ell\left(R /(\underline{y}) p^{n d}-\ell(M) p^{n d}=\ell(K) p^{n d} .\right.
\end{aligned}
$$

The argument above applied to $K$ instead of $M$ then gives that $\operatorname{Tor}_{1}^{R}\left(K, f^{n} R\right)=0$. However, $\operatorname{Tor}_{1}^{R}\left(K,{ }^{n} R\right)=\operatorname{Tor}_{2}^{R}\left(M, f^{n} R\right)$ since $\operatorname{Tor}_{i}^{R}\left((S /(\underline{x}, \underline{y}))^{k}, f^{n} R\right)=0$ for any $i>0$ by Theorem 1.1 .

In the same way one easily sees that $\operatorname{Tor}_{i}^{R}\left(M,{ }^{f^{n}} R\right)=0$ for all $i>0$ and $n \geq 0$, and so by Theorem 1.2 the module $M$ has finite projective dimension. 
In view of [D1] and [D2], the numbers $\ell\left(F^{n}(M)\right) / p^{n d}$ may asymptotically approach $\ell(M)$, but not a priori equal $\ell(M)$. A particular case where this occurs naturally is the following:

Definition 2.2 For an m-primary ideal I of $R$, the limit

$$
\lim _{n \rightarrow \infty} \frac{\ell\left(F^{n}(R / I)\right)}{p^{n d}}
$$

is known as the Hilbert-Kunz multiplicity of I, cf. [K2] and [Mo].

Thus we have the corollary:

Corollary 2.3 Let $R$ be a local Noetherian ring of dimension $d$ which is a complete intersection. Let I be an m-primary ideal of $R$. Then the HilbertKunz multiplicity of I equals the length $\ell(R / I)$ if and only if I has finite projective dimension.

Corollary 2.4 Let $R$ be a local Noetherian ring of dimension $d$ which is a complete intersection. Let $M$ be an R-module of finite length. Then for $i \geq 0$ the sequence of numbers

$$
\frac{\ell\left(\operatorname{Tor}_{i}\left(M, f^{n} R\right)\right)}{p^{n d}}
$$

is nondecreasing.

Proof The case $i=0$ is just a restatement of part b) of Theorem 2.1. For $i>0$, let $\underline{y}$ be a maximal $R$-sequence in the annihilator of $M$. By Theorem 1.1, $\operatorname{Tor}_{i}\left(R /(\underline{y}), f^{n} R\right)=0$ for all $i>0$ and $n>0$. Thus, by replacing $M$ with the $(i-1)$-th syzygy of $M$ over the ring $R /(y)$, we may assume that $i=1$ in the statement to be proved. As before, we get an exact sequence,

$$
0 \rightarrow \operatorname{Tor}_{1}^{R}\left(M,{ }^{f^{n}} R\right) \rightarrow F^{n}(Q) \rightarrow F^{n}\left((R /(\underline{y}))^{k}\right) \rightarrow F^{n}(M) \rightarrow 0
$$

Computing lengths, we get

$$
\frac{\ell\left(\operatorname{Tor}_{1}^{R}\left(M, f^{n} R\right)\right)}{p^{n d}}=\frac{\ell\left(F^{n}(Q)\right)}{p^{n d}}+\frac{\ell\left(F^{n}(M)\right)}{p^{n d}}-\frac{k \cdot \ell\left(F^{n}(R /(\underline{y}))\right)}{p^{n d}}
$$

The first two terms on the right hand side are nondecreasing by part a) of Theorem 2.1, whereas the last is the constant $k \cdot \ell(R /(\underline{y}))$ since $\underline{y}$ is an $R$-sequence. This proves our assertion. 
Corollary 2.5 Let $R$ be a local Noetherian ring of dimension $d$ which is a complete intersection. Let $M$ be an R-module of finite length. If for some $i>0$

$$
\lim _{n \rightarrow \infty} \frac{\ell\left(\operatorname{Tor}_{i}^{R}\left(M,{ }^{n} R\right)\right)}{p^{n d}}=0
$$

then $M$ has finite projective dimension (and thus $\operatorname{Tor}_{i}^{R}\left(M,{ }^{n} R\right)=0$ for all $i>0$ and $n>0$ ).

Proof Again, letting $y$ be a maximal $R$-sequence in the annihilator of $M$ and using the fact that $\operatorname{Tor}_{1}\left(M,{ }^{n} R\right)=0$, we get the equality

$$
\frac{\ell\left(F^{n}(M)\right)}{p^{n d}}+\frac{\ell\left(F^{n}(Q)\right)}{p^{n d}}=\frac{\ell\left(F^{n}\left((R /(\underline{y}))^{k}\right)\right)}{p^{n d}}=\ell(M)+\ell(Q) .
$$

By part b) of Theorem 2.1, the equality $\ell\left(F^{n}(M)\right) / p^{n d}=\ell(M)$ is forced, and so $M$ has finite projective dimension.

\section{Certain one-dimensional Cohen-Macaulay rings and an example}

Using a result of Regnath and Seibert, we will show that Theorem 2.1 holds also for cyclic modules over Cohen-Macaulay rings of dimension one which are geometrically unibranch in the sense of Grothendieck.

Definition 3.1 A local ring $S$ is called geometrically unibranch if the reduced ring $S_{\text {red }}$ is a domain, the integral closure $\overline{S_{\text {red }}}$ of $S_{\text {red }}$ is local, and the residue field extension of $\overline{S_{\text {red }}}$ over $S_{\text {red }}$ is purely inseparable. In particular, a complete local ring with algebraically closed residue field is geometrically unibranch.

We will use the following result of Regnath and Seibert to prove our next result.

Theorem 3.2 ([RS], part of Theorem 1) Let $R$ be a Noetherian local ring of characteristic $p>0$. Suppose that $R$ is geometrically unibranch and has dimension one. Then for any ideal I of $R$, there exists an $n>0$ such that $I^{\left[p^{n}\right]}$ is principal.

Theorem 3.3 Let $R$ be a Cohen-Macaulay local ring of dimension one which is geometrically unibranch. Let I be an m-primary ideal of $R$. Then $R / I$ (or, equivalently, $I$ ) has finite projective dimension if and only if $\lim _{n \rightarrow \infty} \ell\left(F^{n}(R / I)\right) / p^{n}=\ell(R / I)$. 
Proof Suppose first that $\lim _{n \rightarrow \infty} \ell\left(F^{n}(R / I)\right) / p^{n}=\ell(R / I)$. By the result of Regnath and Seibert, for some $n_{0}>0, I^{\left[p^{n_{0}}\right]}$ is principal, say generated by $a$. If $I$ is generated by $f_{1}, \ldots, f_{r}$, then $I^{\left[p^{n}\right]}$ is generated by $f_{1}^{p^{n}}, \ldots, f_{r}^{p^{n}}$. Since $R$ is local, we can take $a=f_{i}^{p^{n}}$ for some $i$. Then for all $n \geq n_{0}$ we have that $I^{\left[p^{n}\right]}=\left(f_{i}^{p^{n}}\right)$ and so we get that

$$
\frac{\ell\left(F^{n}(R / I)\right)}{p^{n}}=\frac{\ell\left(R / I^{\left[p^{n}\right]}\right)}{p^{n}}=\frac{\ell\left(R /\left(f_{i}^{p^{n}}\right)\right)}{p^{n}}=\ell\left(R /\left(f_{i}\right)\right) .
$$

Letting $n$ approach infinity, we get that $\ell(R / I)=\ell\left(R /\left(f_{i}\right)\right)$, but then $\left(f_{i}\right) \subseteq$ $I$ implies that $\left(f_{i}\right)=I$. Therefore $R / I$ has finite projective dimension $\left(f_{i}\right.$ is a non-zero divisor since $R$ is Cohen-Macaulay).

For the converse, see [D1], formula (18).

In fact, the equality $\ell\left(F^{n}(M)\right)=\ell(M) p^{n d}$ holds over Cohen-Macaulay rings of dimension less than or equal to two (see [D1, 1.14]). We next look at an example which shows, on the other hand, that the equality does not hold in general for modules $M$ of finite length and finite projective dimension over Cohen-Macaulay rings. This example is explained in [R2, Chapter 13]. In [R1, Sect. 4], Paul Roberts uses the counterexample of Dutta, Hochster and McLaughlin in [DHM] to construct an example of a module $N$ of finite length 29 and finite projective dimension over a Cohen-Macaulay nonGorenstein ring $R$ of dimension 3 such that the length of $N^{\vee}=\operatorname{Ext}_{R}^{3}(N, R)$ is not equal to the length of $N$. Let $F$. be a free resolution of $N$ over $R$. To show that these lengths are unequal, Roberts determines the values of the local Chern characters $\operatorname{ch}_{i}(F$.) of the complex $F$. applied to the components $\tau_{i}(R)$ of the Todd class $\tau(R)$ of $R$ for each $i=0, \ldots, d$. He obtains that $\operatorname{ch}_{3}(F).\left(\tau_{3}(R)\right)=30, \operatorname{ch}_{2}(F).\left(\tau_{2}(R)\right)=-1, \operatorname{ch}_{1}(F).\left(\tau_{1}(R)\right)=0$, and $\operatorname{ch}_{0}(F).\left(\tau_{0}(R)\right)=0$. The explanation on pp. 429-430 of [R1] shows that

$$
\lim _{n \rightarrow \infty} \frac{\ell\left(F^{n}(N)\right)}{p^{3 n}}=\operatorname{ch}_{3}(F .)=30,
$$

but the local Riemann-Roch Theorem formula for the Euler characteristic $\chi(F$.) states that

$$
\ell(N)=\chi(F .)=\operatorname{ch}(F .)(\tau(R))=\sum_{i=0}^{3} \operatorname{ch}_{i}(F .)\left(\tau_{i}(R)\right)=29 .
$$

So, clearly

$$
\lim _{n \rightarrow \infty} \frac{\ell\left(F^{n}(N)\right)}{p^{3 n}} \neq \ell(N) .
$$

For Gorenstein domains of dimension less than or equal to three, the equality does hold for modules of finite length and finite projective dimension (see [D1]), but it has recently been shown not to hold in general over Gorenstein rings [MS]. 
Acknowledgements. We are grateful to Sankar Dutta for many helpful discussions along the way. We also thank Gebhard Boeckle for his help with the equivalence of conditions ii) and iii) of Theorem 2.1, as well as the referee for suggestions improving the presentation of the results.

\section{References}

[D1] Dutta, S.P.: Frobenius and multiplicities. J. Algebra 85, 424-448 (1983)

[D2] Dutta, S.P.: Ext and Frobenius. J. Algebra 127, 163-177 (1989)

[DHM] Dutta, S.P., Hochster, M., McLaughlin, J.E.: Modules of finite projective dimension with negative intersection multiplicities. Invent. Math. 79, 253-291 (1985)

[He] Herzog, J.: Ringe der Charakteristik $p$ und Frobenius-Funktoren. Math. Z. 140, 67-78 (1974)

[K1] Kunz, E.: Characterization of regular local rings of characteristic $p$. Amer. J. Math. 41, 772-784 (1969)

[K2] Kunz, E.: On Noetherian rings of characteristic p. Amer. J. Math. 98, 999-1013 (1976)

[MS] Miller, C., Singh, A.: Intersection multiplicities over Gorenstein rings, preprint.

[Mo] Monsky, P.: The Hilbert-Kunz function. Math. Ann. 263, 43-49 (1983)

[PS] Peskine, C., Szpiro, L.: Dimension projective finie et cohomologie locale. Inst. Haut. Études Sci. Publ. Math. 42, 47-119 (1973)

[RS] Regnath, H., Seibert, G.: Frobenius functors and invariant factors. Comm. Algebra 26, 1757-1768 (1998)

[R1] Roberts, P.: Intersection theorems. In: Hochster, M. et al. (eds.): Commutative Algebra. Proceedings of an MSRI Microprogram (Math. Sci. Research Inst. Publ., vol. 15) pp. 417-436. Berlin Heidelberg New York: Springer 1989

[R2] Roberts, P.: Multiplicities and Chern Classes in Local Algebra (Cambridge Tracts in Mathematics, No 133) Cambridge: Cambridge University Press 1998 